\title{
Continuous Wavelet Transform in Quantum Field Theory
}

\author{
M.V.Altaisky \\ Space Research Institute RAS, Profsoyuznaya 84/32, Moscow, 117997, Russia* \\ N.E.Kaputkina \\ National Technological University "MISiS", Leninsky prospect 4, Moscow, 119049, Russia
}

(Dated: revised June 15, 2013)

\begin{abstract}
We describe the application of the continuous wavelet transform to calculation of the Green functions in quantum field theory: scalar $\phi^{4}$ theory, quantum electrodynamics, quantum chromodynamics. The method of continuous wavelet transform in quantum field theory presented in [1] for the scalar $\phi^{4}$ theory, consists in substitution of the local fields $\phi(x)$ by those dependent on both the position $x$ and the resolution $a$. The substitution of the action $S[\phi(x)]$ by the action $S\left[\phi_{a}(x)\right]$ makes the local theory into nonlocal one, and implies the causality conditions related to the scale $a$, the region causality [2]. These conditions make the Green functions $G\left(x_{1}, a_{1}, \ldots, x_{n}, a_{n}\right)=\left\langle\phi_{a_{1}}\left(x_{1}\right) \ldots \phi_{a_{n}}\left(x_{n}\right)\right\rangle$ finite for any given set of regions by means of an effective cutoff scale $A=\min \left(a_{1}, \ldots, a_{n}\right)$.

PACS numbers: 03.70.+k, 11.10.-z
\end{abstract}

\section{INTRODUCTION}

The fundamental problem of quantum field theory and statistical mechanics is the problem of divergences of Feynman integrals emerging in Green functions. The formal infinities appearing in perturbation expansion of Feynman integrals are tackled with different regularization methods, from Pauli-Villars regularization to renormalization methods for gauge theories, see e.g. [3] for a review. A special class of regularizations are the lattice regularizations tailored for the precise numerical simulations in gauge theories $[4,5]$.

There are a few basic ideas connected with those regularizations. First, certain minimal scale $L=\frac{2 \pi}{\Lambda}$, where $\Lambda$ is the cutoff momentum, is introduced into the theory, with all the fields $\phi(x)$ being substituted by their Fourier transforms truncated at momentum $\Lambda$. The physical quantities are then demanded to be independent on the rescaling of the cut-off parameter $\Lambda$. The second thing is the Kadanoff blocking procedure [6], which averages the small-scale fluctuations up to a certain scale this makes a kind of effective interaction.

Physically all these methods imply to the selfsimilarity assumption: blocks interact to each other similarly to the sub-blocks [7]. Similarly, but not necessarily having the same interaction strength - the latter can be dependent on scale $\lambda=\lambda(a)$. However there is no place for such dependence if the fields are described solely in terms of their Fourier transform - except for the cutoff momentum dependence. The latter representation, being based on the representation of the translation group, is

\footnotetext{
*Electronic address: altaisky@mx.iki.rssi.ru; Also at Bogoliubov Laboratory of Theoretical Physics, Joint Institute for Nuclear Research, Dubna, 141980, Russia

${ }^{\dagger}$ Electronic address: nataly@misis.ru
}

rather restrictive: it determines the effective interaction of all fluctuations up to a certain scale, but says nothing about the interaction of the fluctuations at a given scale.

That is why, the functional methods capable of taking into account the interaction at specific scale are required. Wavelet analysis, being the multiscale alternative to the Fourier transform, emerged in geophysics [8], is the most known of such methods. Its application to quantum field theory have been suggested by many authors [9-13] The other side of the problem is that the quantum nature of the fields considered in quantum field theory is constrained by the Heisenberg uncertainty principle. To localize a particle in an interval $\Delta x$ the measuring device requests a momentum transfer of order $\Delta p \sim \hbar / \Delta x$. If $\Delta x$ is too small the field $\phi(x)$ at a fixed point $x$ has no experimentally verifiable meaning. What is meaningful, is the vacuum expectation of product of fields in certain region centered around $x$, the width of which $(\Delta x)$ is constrained by the experimental conditions of the measurement [1]. That is why, at least from physical point of view, any such field should be designated by the resolution of observation $\phi_{\Delta x}(x)$.

In present paper we exploit the observation that quantum field theory models, which yield divergent Feynman graphs, can be studied analytically if we project original fields $\phi(x)$ into the fields $\phi_{a}(x)$, subscribed by the scale of measurement $a$. The Green functions $\left\langle\phi_{a_{1}}\left(x_{1}\right) \ldots \phi_{a_{n}}\left(x_{n}\right)\right\rangle$ become finite under certain causality assumptions, which stand for the fact that any $n$-point correlation function can be dependent only on space-time regions, rather than points, and thus cannot be infinite [1]. These Green function describe the effect of propagation of a perturbation from a region of size $a$, centered at a point $x$, to a region of size $a^{\prime}$, centered at a point $x^{\prime}$. The standard quantum field theory models can be reformulated by expressing the (point-dependent) local fields $\phi(x)$, the distributions, in terms of the regiondependent fields $\phi_{a}(x)$. The integration over all scales $a$ 
will of course drive us back to the known divergent results, but the physical observables are always those measured with finite resolution, and their correlations are always finite. Therefore the idea of wavelet transform of quantum fields, which will be considered below, is very similar to the idea of renormalization group (This similarity, being studied in the lattice framework $[12,14]$, is out of coverage of the present paper).

The remainder of this paper is organized as follows. In Section $I I$ we recall the basics of the continuous wavelet transform and its application to the multiresolution analysis of quantum fields. The definitions of scale-dependent fields and Green functions, the modifications of the Feynman diagram technique are presented. The $\phi^{4}$ scalar field model examples of calculations are given. Section III considers the case of operator-valued scale-dependent fields. The operator ordering and commutation relations are presented. The relations between the theory of scaledependent fields in Euclidean and Minkowski spaces are discussed. Section IV presents the examples of calculation of one-loop Feynman graphs in QED and QCD. The Conclusion gives a few remarks on the perspectives and applicability of the multiscale field theory approach based on continuous wavelet transform.

\section{CONTINUOUS WAVELET TRANSFORM IN QUANTUM FIELD THEORY}

\section{A. Basics of the continuous wavelet transform}

Let $\mathcal{H}$ be a Hilbert space of states for a quantum field $|\phi\rangle$. Let $G$ be a locally compact Lie group acting transitively on $\mathcal{H}$, with $d \mu(\nu), \nu \in G$ being a left-invariant measure on $G$. Then, similarly to representation of a vector $|\phi\rangle$ in a Hilbert space of states $\mathcal{H}$ as a linear combination of an eigenvectors of momentum operator $|\phi\rangle=\int|p\rangle d p\langle p \mid \phi\rangle$, any $|\phi\rangle \in \mathcal{H}$ can be decomposed with respect to a representation $U(\nu)$ of $G$ in $\mathcal{H}[15,16]$ :

$$
|\phi\rangle=\frac{1}{C_{g}} \int_{G} U(\nu)|g\rangle d \mu(\nu)\left\langle g\left|U^{*}(\nu)\right| \phi\right\rangle,
$$

where $|g\rangle \in \mathcal{H}$ is referred to as an admissible vector, or basic wavelet, satisfying the admissibility condition

$$
C_{g}=\frac{1}{\|g\|^{2}} \int_{G}|\langle g|U(\nu)| g\rangle|^{2} d \mu(\nu)<\infty .
$$

The coefficients $\left\langle g\left|U^{*}(\nu)\right| \phi\right\rangle$ are referred to as wavelet coefficients.

If the group $G$ is abelian, the wavelet transform (1) with $G: x^{\prime}=x+b^{\prime}$ coincides with Fourier transform.

The next to the abelian group is the group of the affine transformations of the Euclidean space $\mathbb{R}^{d}$

$$
G: x^{\prime}=a R(\theta) x+b, x, b \in \mathbb{R}^{d}, a \in \mathbb{R}_{+}, \theta \in S O(d),
$$

where $R(\theta)$ is the rotation matrix. We define unitary representation of the affine transform (2) with respect to the basic wavelet $g(x)$ as follows:

$$
U(a, b, \theta) g(x)=\frac{1}{a^{d}} g\left(R^{-1}(\theta) \frac{x-b}{a}\right) .
$$

(We use $L^{1}$ norm $[17,18]$ instead of usual $L^{2}$ to keep the physical dimension of wavelet coefficients equal to the dimension of the original fields).

Thus the wavelet coefficients of the function $\phi(x) \in$ $L^{2}\left(\mathbb{R}^{d}\right)$ with respect to the basic wavelet $g(x)$ in $\mathrm{Eu}-$ clidean space $\mathbb{R}^{d}$ can be written as

$$
\phi_{a, \theta}(b)=\int_{\mathbb{R}^{d}} \frac{1}{a^{d}} \overline{\left(R^{-1}(\theta) \frac{x-b}{a}\right)} \phi(x) d^{d} x .
$$

The wavelet coefficients (4) represent the result of the measurement of function $\phi(x)$ at the point $b$ at the scale $a$ with an aperture function $g$ rotated by the angle(s) $\theta$ [19].

The function $\phi(x)$ can be reconstructed from its wavelet coefficients (4) using the formula (1):

$$
\phi(x)=\frac{1}{C_{g}} \int \frac{1}{a^{d}} g\left(R^{-1}(\theta) \frac{x-b}{a}\right) \phi_{a \theta}(b) \frac{d a d^{d} b}{a} d \mu(\theta)
$$

The normalization constant $C_{g}$ is readily evaluated using Fourier transform:

$$
C_{g}=\int_{0}^{\infty}\left|\tilde{g}\left(a R^{-1}(\theta) k\right)\right|^{2} \frac{d a}{a} d \mu(\theta)=\int|\tilde{g}(k)|^{2} \frac{d^{d} k}{|k|^{d}}<\infty .
$$

For isotropic wavelets

$$
C_{g}=\int_{0}^{\infty}|\tilde{g}(a k)|^{2} \frac{d a}{a}=\int|\tilde{g}(k)|^{2} \frac{d^{d} k}{S_{d}|k|^{d}},
$$

where $S_{d}=\frac{2 \pi^{d / 2}}{\Gamma(d / 2)}$ is the area of unit sphere in $\mathbb{R}^{d}$.

\section{B. Resolution-dependent fields}

If the ordinary quantum field theory defines the field function $\phi(x)$ as a scalar product of the state vector of the system and the state vector corresponding to the localization at the point $x$ :

$$
\phi(x) \equiv\langle x \mid \phi\rangle,
$$

the modified theory $[1,20]$ should respect the resolution of the measuring equipment. Namely, we define the resolution-dependent fields

$$
\phi_{a \theta}(x) \equiv\langle x, \theta, a ; g \mid \phi\rangle,
$$

also referred to as the scale components of $\phi$, where $\langle x, \theta, a ; g|$ is the bra-vector corresponding to localization of the measuring device around the point $x$ with the spatial resolution $a$ and the orientation $\theta \in S O(d) ; g$ labels the apparatus function of the equipment, an aperture [19]. 
The field theory of extended objects with the basis $g$ defined on the spin variables was considered in [21, 22].

The introduction of resolution into the definition of the field function has a clear physical interpretation. If the particle, described by the field $\phi(x)$, have been initially prepared in the interval $\left(x-\frac{\Delta x}{2}, x+\frac{\Delta x}{2}\right)$, the probability of registering this particle in this interval is generally less than unity: for the probability of registration depends on the strength of interaction and the ratio of typical scales of the measured particle and the measuring equipment. The maximum probability of registering an object of typical scale $\Delta x$ by the equipment with typical resolution $a$ is achieved when these two parameters are comparable. For this reason the probability of registering an electron by visual range photon scattering is much higher than by that of long radio-frequency waves. As mathematical generalization, we should say that if a measuring equipment with a given spatial resolution $a$ fails to register an object, prepared on spatial interval of width $\Delta x$ with certainty, then tuning the equipment to all possible resolutions $a^{\prime}$ would lead to the registration. This certifies the fact of the existence of the measured object.

In terms of the resolution-dependent field (7) the unit probability of registering the object $\phi$ anywhere in space at any resolution and any orientation of the measuring device is expressed by normalization

$$
\int\left|\phi_{a, \theta}(x)\right|^{2} d \mu(a, \theta, x)=1,
$$

where $d \mu(a, \theta, x)$ is an invariant measure on $\mathbb{R}_{+} \times S O(d) \times$ $\mathbb{R}^{d}$, which depends on the position $x$, the resolution $a$, and the orientation $\theta$ of the aperture $g$.

If the measuring equipment has the resolution $A$, i.e. all states $\langle g ; a \geq A, x \mid \phi\rangle$ are registered with significant probability, but those with $a<A$ are not, the regularization of the field theory in momentum space, with the cutoff momentum $\Lambda=2 \pi / A$ corresponds to the UVregularized functions

$$
\phi^{(A)}(x)=\frac{1}{C_{g}} \int_{a \geq A}\langle x \mid g ; a, b\rangle d \mu(a, b)\langle g ; a, b \mid \phi\rangle .
$$

The regularized $n$-point Green functions are $\mathcal{G}^{(A)}\left(x_{1}, \ldots, x_{n}\right) \equiv\left\langle\phi^{(A)}\left(x_{1}\right), \ldots, \phi^{(A)}\left(x_{n}\right)\right\rangle_{c}$.

However, the momentum cutoff is merely a technical trick: the physical analysis, performed by renormalization group method [3, 23, 24], demands the independence of physical results from the cutoff at $\Lambda \rightarrow \infty$.

\section{Scalar field example}

To illustrate the method, following $[1,20]$, we start with Euclidean scalar field theory. The widely known example which fairly illustrates the problem is the $\phi^{4}$ interaction model in $\mathbb{R}^{d}$, see e.g. $[3,25]$, determined by the generating functional

$$
W[J]=\mathcal{N} \int e^{-\int d^{d} x\left[\frac{1}{2}(\partial \phi)^{2}+\frac{m^{2}}{2} \phi^{2}+\frac{\lambda}{4 !} \phi^{4}-J \phi\right]} \mathcal{D} \phi,
$$

where $\mathcal{N}$ is a formal normalization constant. The connected Green functions are given by variational derivatives of the generating functional:

$$
G^{(n)}=\left.\frac{\delta^{n} \ln W[J]}{\delta J\left(x_{1}\right) \ldots \delta J\left(x_{n}\right)}\right|_{J=0} .
$$

In statistical sense these functions have the meaning of the $n$-point correlation functions [26]. The divergences of Feynman graphs in the perturbation expansion of the Green functions (11) with respect to the coupling constant $\lambda$ emerge at coinciding arguments $x_{i}=x_{k}$. For instance, the bare two-point correlation function

$$
G_{0}^{(2)}(x-y)=\int \frac{d^{d} p}{(2 \pi)^{d}} \frac{e^{-\imath p(x-y)}}{p^{2}+m^{2}}
$$

is divergent at $x=y$ for $d \geq 2$.

For simplicity let us assume the basic wavelet $g$ to be isotropic, i.e. we can drop the rotation matrix $R(\theta)$. Substitution of the continuous wavelet transform (5) into field theory (10) gives the generating functional for the scale-dependent fields $\phi_{a}(x)[20]$ :

$$
\begin{aligned}
& W_{W}\left[J_{a}\right]= \\
& \mathcal{N} \int \mathcal{D} \phi_{a}(x) \exp \left[-\frac{1}{2} \int \phi_{a_{1}}\left(x_{1}\right) D\left(a_{1}, a_{2}, x_{1}-x_{2}\right) \phi_{a_{2}}\left(x_{2}\right) \frac{d a_{1} d^{d} x_{1}}{a_{1}} \frac{d a_{2} d^{d} x_{2}}{a_{2}}\right. \\
&\left.-\frac{\lambda}{4 !} \int V_{x_{1}, \ldots, x_{4}}^{a_{1}, \ldots, a_{4}} \phi_{a_{1}}\left(x_{1}\right) \cdots \phi_{a_{4}}\left(x_{4}\right) \frac{d a_{1} d^{d} x_{1}}{a_{1}} \frac{d a_{2} d^{d} x_{2}}{a_{2}} \frac{d a_{3} d^{d} x_{3}}{a_{3}} \frac{d a_{4} d^{d} x_{4}}{a_{4}}+\int J_{a}(x) \phi_{a}(x) \frac{d a d^{d} x}{a}\right]
\end{aligned}
$$

with $D\left(a_{1}, a_{2}, x_{1}-x_{2}\right)$ and $V_{x_{1}, \ldots, x_{4}}^{a_{1}, \ldots, a_{4}}$ denoting the wavelet images of the inverse propagator and that of the interaction potential. The Green functions for scale component fields are given by functional derivatives

$$
\left\langle\phi_{a_{1}}\left(x_{1}\right) \cdots \phi_{a_{n}}\left(x_{n}\right)\right\rangle_{c}=\left.\frac{\delta^{n} \ln W_{W}\left[J_{a}\right]}{\delta J_{a_{1}}\left(x_{1}\right) \ldots \delta J_{a_{n}}\left(x_{n}\right)}\right|_{J=0} .
$$

Surely the integration in (13) over all scale variables $\int_{0}^{\infty} \frac{d a_{i}}{a_{i}}$ turns us back to the divergent theory (10).

This is the point to restrict the functional integration in (13) only to the field configurations $\left\{\phi_{a}(x)\right\}_{a \geq A}$. The restriction is imposed at the level of the Feynman diagram technique. Indeed, applying the Fourier transform 
to the r.h.s. of $(5,4)$ one yields

$$
\begin{aligned}
\phi(x) & =\frac{1}{C_{g}} \int_{0}^{\infty} \frac{d a}{a} \int \frac{d^{d} k}{(2 \pi)^{d}} e^{-\imath k x} \tilde{g}(a k) \tilde{\phi}_{a}(k), \\
\tilde{\phi}_{a}(k) & =\frac{\tilde{g}(a k)}{\phi}(k) .
\end{aligned}
$$

Doing so, we have the following modification of the Feynman diagram technique [13]:

- each field $\tilde{\phi}(k)$ will be substituted by the scale component $\tilde{\phi}(k) \rightarrow \tilde{\phi}_{a}(k)=\overline{\tilde{g}(a k)} \tilde{\phi}(k)$.

- each integration in momentum variable is accompanied by corresponding scale integration:

$$
\frac{d^{d} k}{(2 \pi)^{d}} \rightarrow \frac{d^{d} k}{(2 \pi)^{d}} \frac{d a}{a} .
$$

- each interaction vertex is substituted by its wavelet transform; for the $N$-th power interaction vertex this gives multiplication by factor $\prod_{i=1}^{N} \overline{\tilde{g}\left(a_{i} k_{i}\right)}$.

According to these rules the bare Green function in wavelet representation takes the form

$$
G_{0}^{(2)}\left(a_{1}, a_{2}, p\right)=\frac{\tilde{g}\left(a_{1} p\right) \tilde{g}\left(-a_{2} p\right)}{p^{2}+m^{2}} .
$$

The finiteness of the loop integrals is provided by the following rule: there should be no scales $a_{i}$ in internal lines smaller than the minimal scale of all external lines. Therefore the integration in $a_{i}$ variables is performed from the minimal scale of all external lines up to the infinity.

To understand how the method works one can look at the one-loop contributions to the two-point Green function $G^{(2)}\left(a_{1}, a_{2}, p\right)$ shown in Fig. 1a., and to the vertex shown in Fig. 1b. The best choice of the wavelet function

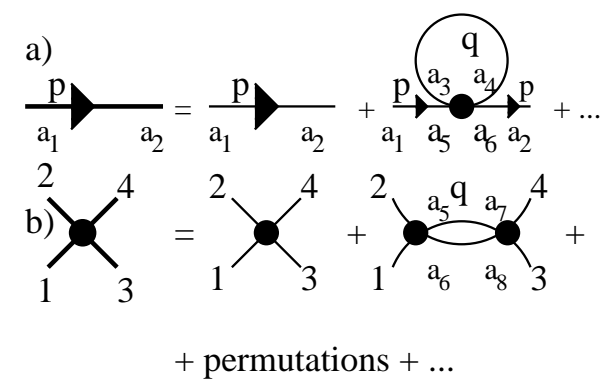

FIG. 1: Feynman diagrams for the Green functions $G^{(2)}$ and $G^{(4)}$ for the resolution-dependent fields. Redrawn from [1]

$g(x)$ would be the apparatus function of the measuring device, however any well localized function with $\tilde{g}(0)=0$ will suit. The tadpole integral, to keep with the notation of [20], is written as

$$
\begin{aligned}
& T_{1}^{d}(A m)=\frac{1}{C_{g}^{2}} \int_{a_{3}, a_{4} \geq A} \frac{d^{d} q}{(2 \pi)^{d}} \frac{\left|\tilde{g}\left(a_{3} q\right)\right|^{2}\left|\tilde{g}\left(-a_{4} q\right)\right|^{2}}{q^{2}+m^{2}} \frac{d a_{3}}{a_{3}} \frac{d a_{4}}{a_{4}} \\
&= \\
& \frac{S_{d} m^{d-2}}{(2 \pi)^{d}} \int_{0}^{\infty} f^{2}(A m x) \frac{x^{d-1} d x}{x^{2}+1}
\end{aligned}
$$

where the integration over the scale variables resulted in the effective cutoff function

$$
f(x) \equiv \frac{1}{C_{g}} \int_{x}^{\infty}|\tilde{g}(a)|^{2} \frac{d a}{a}, \quad f(0)=1
$$

which depends on the squared modulus of the Fourier image of the basic wavelet, and thus is even with respect to reflections.

In the one-loop contribution to the vertex, shown in Fig. 1b, the value of the loop integral is

$$
X_{d}=\frac{\lambda^{2}}{2} \frac{1}{(2 \pi)^{d}} \int \frac{d^{d} q}{(2 \pi)^{d}} \frac{f^{2}(q A) f^{2}((q-s) A)}{\left[q^{2}+m^{2}\right]\left[(q-s)^{2}+m^{2}\right]},
$$

where $s=p_{1}+p_{2}, A=\min \left(a_{1}, a_{2}, a_{3}, a_{4}\right)$. The integral (15) can be calculated by symmetrization of loop momenta $q \rightarrow q+\frac{s}{2}$ in Fig. 1b, introducing dimensionless variable $\mathbf{y}=\mathbf{q} / s$, after a simple algebra we get

$$
\begin{array}{r}
X_{d}=\frac{\lambda^{2}}{2} \frac{S_{d-1} s^{d-4}}{(2 \pi)^{2 d}} \int_{0}^{\pi} d \theta \sin ^{d-2} \theta \int_{0}^{\infty} d y y^{d-3} \times \\
\frac{f^{2}\left(A s \sqrt{y^{2}+y \cos \theta+\frac{1}{4}}\right) f^{2}\left(A s \sqrt{y^{2}-y \cos \theta+\frac{1}{4}}\right)}{\left[\frac{y^{2}+\frac{1}{4}+\frac{m^{2}}{s^{2}}}{y}+\cos \theta\right]\left[\frac{y^{2}+\frac{1}{4}+\frac{m^{2}}{s^{2}}}{y}-\cos \theta\right]}
\end{array}
$$

where $\theta$ is the angle between the loop momentum $q$ and the total momentum $s$. For the simple choice of the basic wavelet $g_{1}[1,20]$

$$
g(x)=-\frac{x e^{-x^{2} / 2}}{(2 \pi)^{d / 2}}, \quad \tilde{g}(k)=\imath k e^{-k^{2} / 2}
$$

in four dimensions we get a finite result

$$
\begin{array}{rlr}
T_{1}^{4}\left(\alpha^{2}\right) & = & \frac{-4 \alpha^{4} e^{2 \alpha^{2}} \operatorname{Ei}_{1}\left(2 \alpha^{2}\right)+2 \alpha^{2}}{64 \pi^{2} \alpha^{4}} m^{2} \\
\lim _{s^{2} \gg 4 m^{2}} X_{4}\left(\alpha^{2}\right) & =\frac{\lambda^{2}}{256 \pi^{6}} \frac{e^{-2 \alpha^{2}}}{2 \alpha^{2}}\left[e^{\alpha^{2}}-1-\alpha^{2} e^{2 \alpha^{2}} \operatorname{Ei}_{1}\left(\alpha^{2}\right)\right. \\
+ & \left.2 \alpha^{2} e^{2 \alpha^{2}} \operatorname{Ei}_{1}\left(2 \alpha^{2}\right)\right]
\end{array}
$$

depending on is dimensionless scale factor $\alpha \equiv A m$, where $A$ is the minimal scale of all external lines.

These results display an evident fact that for the massive scalar field all length scales are to be measured in the units of inverse mass. 


\section{CAUSALITY AND COMMUTATION RELATIONS}

\section{A. Operator ordering}

Up to now we have considered the calculation of the Feynman diagrams for the scale-dependent fields $\phi_{a, .}(x)$ treated as $c$-valued functions. In quantum field theory, adjusted to high energy physics applications, the fields $\phi_{a, .}(x)$ are operator-valued functions. So, as it was already emphasized in the context of the wavelet application to quantum chromodynamics $[1,11]$, the operator ordering and the commutation relations are to be defined.

In standard quantum field theory the operator ordering is performed according to the non-decreasing of the time argument in the product of the operator-valued functions acting on vacuum state

$$
\underbrace{A\left(t_{n}\right) A\left(t_{n-1}\right) \ldots A\left(t_{2}\right) A\left(t_{1}\right)}_{t_{n} \geq t_{n-1} \geq \ldots \geq t_{2} \geq t_{1}}|0\rangle .
$$

In the infinite momentum frame (IMF), which simplifies algebraic structure of the current algebra, the timeordering is performed in the proper time argument $x_{+}$ [27]. The quantization is performed by separating the Fourier transform of quantum fields into the positive- and the negative-frequency parts

$$
\phi=\phi^{+}(x)+\phi^{-}(x),
$$

defined as follows

$$
\phi(x)=\int \frac{d^{d} k}{(2 \pi)^{d}}\left[e^{\imath k x} u^{+}(k)+e^{-\imath k x} u^{-}(k)\right],
$$

where the operators $u^{ \pm}(k)=u( \pm k) \theta\left(k_{0}\right)$ are subjected to canonical commutation relations

$$
\left[u^{+}(k), u^{-}\left(k^{\prime}\right)\right]=\Delta\left(k, k^{\prime}\right)
$$

In case of the scale-dependent fields, because of the presence of the scale argument in new fields $\phi_{a, \eta}(x)$, where $a$ and $\eta$ label the size and the shape of the region centered at $x$, the problem arises how to order the operators supported by different regions. This problem was solved in $[1,28]$ on the base of the region causality assumption [2]. If two regions $(\Delta x, x)$ and $(\Delta y, y)$ do not intersect the standard time ordering procedure is applied. Alternatively, if one of the regions is inside another, see Fig. 2 the operator standing for the bigger region acts on vacuum first [1]. This causal ordering, drawn in Euclidean space, is presented in Fig. 2 below. The time ordering in Euclidean space, as an analytic continuation of time ordering in Minkowski space, have been already considered in [29]. The diagram Fig. 2 shows space-like regions in Euclidean space. For Minkowski space corresponding diagrams can be obtained by analytic continuation of the Euclidean ball of imaginary radius $\imath \Delta$ into Minkowski space, where we can restrict ourselves with forward light
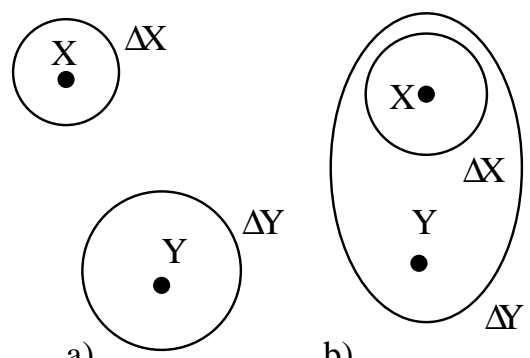

b)

FIG. 2: Causal ordering of scale-dependent fields. Space-like regions are drawn in Euclidean space: a) The event regions do not intersect; b) Event $X$ is inside the event $Y$.

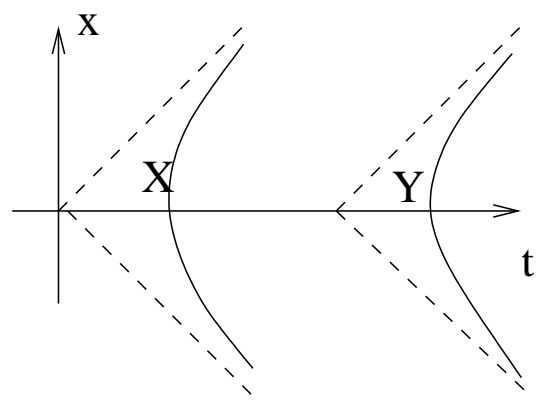

FIG. 3: Disjoint events in $(t, x)$ plane in Minkowski space

cone $t \geq 0,|x| \leq t$. The disjoint events in Minkowski space are shown in Fig. 3. The correspondence to the other case of one Euclidean event inside another, shown in Fig. 2b, looks more complex after analytic continuation to Minkowski space. The forward light-cone part of such intersection is shown in Fig. 4.

We consider partial intersection of regions $(A \cap B=$ $C, C \neq A, C \neq B, C \neq \emptyset)$ as unphysical. For this reason corresponding ordering of operator-valued fields is not defined. Since a region is identified with a possibility of measurement, a simultaneous measurement of a part within and not within the parent entity is inconsistent. The "partial intersection" just implies that doing the functional integration one has to go to the finer scale, so that the regions do not intersect. The same happens in $p$-adic models of quantum gravity: two $p$-adic balls are either disjoint or one within another [30].

Mathematically, when we make the functional measure of a Feynman integral into a a discrete product of wavelet fields on a lattice $\mathcal{D} u_{a}(b) \rightarrow \prod_{j, k} d d_{k}^{j}$ we get rid of partial intersection, as it can be seen in the example of a binary tree, shown in the Table I below

Phenomenologically, the principle 'the coarse acts first' is related to the definition of the measurement procedure, possibly generalized, where the state of a part can be measured/affected only after and relatively to the state of the whole. The similar reason underlies the restriction on the scales in internal loops by the minimal scales of all external lines of the Feynman diagram: if we measure a quantum system from outside we cannot excite modes 


\begin{tabular}{|c|c|}
\hline$d_{0}^{0}$ & $d_{1}^{0}$ \\
\hline $1 d_{0}^{1}$ & \\
\hline
\end{tabular}

TABLE I: Binary tree of operator-valued functions. Vertical direction corresponds to the scale variable. The causal sequence of the operator-valued functions shown in the table above is: $d_{0}^{0}, d_{00}^{1}, d_{01}^{1}, d_{1}^{0}, d_{10}^{1}, d_{11}^{1}$. As it is shown the underlined regions of different scales do not intersect

finer than the minimal available scale of measurement. Thus the functional integration over the trajectories in

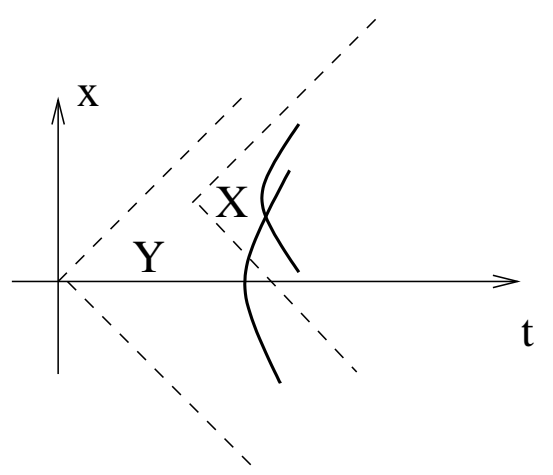

FIG. 4: Nontrivial intersection of two events $X \subset Y$ in $(t, x)$ plane in Minkowski space

the space of square-integrable functions $\mathcal{D} \phi(x)$ is substituted by functional integration over all causal paths, or tubes of all different thickness, in the space of scaledependent functions $\mathcal{D} \phi_{a}(x)$. Referring the reader to the original works of $[2,31]$ for the topological aspects of causal paths we ought mention that the Bogolioubov microcausality condition holds for causal paths in the same way as it holds for trajectories [1]. It is also easy to show, that if the domain $Y$ is inside the domain $X$ the corresponding Green function is not singular at coinciding arguments - it is a projection from coarser scale to finer scale:

$G_{0}^{(2)}\left(a_{1}, a_{2}, b_{1}-b_{2}=0\right)=\int \frac{d^{4} p}{(2 \pi)^{4}} \frac{\tilde{g}\left(a_{1} p\right) \tilde{g}\left(-a_{2} p\right)}{p^{2}+m^{2}} e^{-\imath p \cdot 0}$ since $|\tilde{g}(p)|$ vanish at $p \rightarrow \infty$.

\section{B. Commutation relations}

In case of wavelet transform the positive- and negativefrequency part operators (16) can be expressed using wavelet transform

$$
u_{i}^{ \pm}(k)=\frac{1}{C_{g_{i}}} \int_{-\infty}^{\infty} d \eta \int_{0}^{\infty} \frac{d a}{a} \tilde{g}_{i}\left(a M^{-1}(\eta) k\right) u_{i a \eta}^{ \pm}(k),
$$

from where we can set [1]:

$$
\begin{array}{rr}
{\left[u_{i a \eta}^{+}(k), u_{j a^{\prime} \eta^{\prime}}^{-}\left(k^{\prime}\right)\right]=} & \delta_{i j} C_{g_{i}} a \delta\left(a-a^{\prime}\right) \delta\left(\eta-\eta^{\prime}\right) \times \\
\times & {\left[u^{+}(k), u^{-}\left(k^{\prime}\right)\right]}
\end{array}
$$

to ensure canonical commutation relations for $\left[u^{+}(k), u^{-}\left(k^{\prime}\right)\right]$.

\section{The Dyson-Schwinger equation}

Ordering in scale argument results in the modification of the Dyson-Schwinger equation in the theory of scaledependent functions. Let $G\left(x-y, a_{x}, a_{y}\right)$ be the bare field propagator, describing the propagation of the field from the region $\left(y, a_{y}\right)$ to the region $\left(x, a_{x}\right)$. Let $\mathcal{G}\left(x-y, a_{x}, a_{y}\right)$ be the full propagator for the same regions. The DysonSchwinger equation relating the full propagator with the bare propagator is symbolically drawn in the diagram

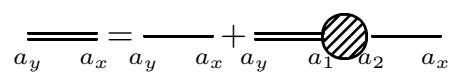

The integral equation depicted in diagram (19) can be written as

$$
\mathcal{G}\left(x-y, a_{x}, a_{y}\right)=G\left(x-y, a_{x}, a_{y}\right)+\int \frac{d a_{1}}{a_{1}} \int \frac{d a_{2}}{a_{2}} \int d x_{1} d x_{2} G\left(x-x_{2}, a_{x}, a_{2}\right) \mathcal{P}\left(x_{2}-x_{1}, a_{2}, a_{1}\right) \mathcal{G}\left(x_{1}-y, a_{1}, a_{y}\right)
$$

where the full vertex $=\mathcal{P}\left(x_{2}-x_{1}, a_{2}, a_{1}\right)$ denotes the vacuum polarization operator if $G$ is the massless boson, or the self-energy diagram otherwise. The Fourier counterpart of the equation (20) can be written as

$$
\tilde{\mathcal{G}}_{a_{x}, a_{y}}(p)=\tilde{G}_{a_{x}, a_{y}}(p)+\int \frac{d a_{1}}{a_{1}} \int \frac{d a_{2}}{a_{2}} \tilde{G}_{a_{x}, a_{2}}(p) \tilde{\mathcal{P}}_{a_{2}, a_{1}}(p) \tilde{\mathcal{G}}_{a_{1}, a_{y}}(p) .
$$




\section{Wavelet transform in Minkowski space}

The straightforward application of wavelet transform (4), defined in Euclidean space $\mathbb{R}^{d}$, to the Minkowski space $M_{1}^{4}$ would be the analytic continuation of the results into the imaginary time $x_{4}=\imath x_{0}$, making the Euclidean rotations into Lorentz boosts. The construction of such wavelets with respect to the representations of the Poincare group have been studied by several authors $[32,33]$. From physical point of view there exists a simple and an elegant way of making wavelet transform in Minkowski space.

In quantum field theory problems related to relativistic particle collisions we can always change the coordinate frame to the co-moving frame of a relativistic projectile moving at utmost speed of light. Due to the Lorentz contraction of the projectile the longitudinal and the transversal degrees of freedom behave essentially different in such system. Without loss of generality we can always assume the projectile to move along the $z$ axis.

The Lorentz contraction, i.e., the hyperbolic rotation in $(t, z)$ plane is determined by the hyperbolic rotation angle - the rapidity $\eta$. The rotations in the transverse plane are not affected by the Lorentz contraction and are determined by the $S O(2)$ rotation angle $\phi$. If the problem is axially symmetric, the latter can be dropped.

Therefore it is convenient to change from the spacetime coordinates $(t, x, y, z)$ to the light-cone coordinates $\left(x_{+}, x_{-}, x, y\right)$ :

$$
x_{ \pm}=\frac{t \pm z}{\sqrt{2}}, \quad \mathbf{x}_{\perp}=(x, y) .
$$

This is the so-called infinite momentum frame. The IMF is not a Lorentzian system, but a limit of that at $v \rightarrow c$. The advantage of the coordinates (21) for the calculations, say in QED or QCD, is significant simplification of the vacuum structure $[27,34]$. The metrics in the lightcone coordinates becomes

$$
g_{\mu \nu}=\left(\begin{array}{cccc}
0 & 1 & 0 & 0 \\
1 & 0 & 0 & 0 \\
0 & 0 & -1 & 0 \\
0 & 0 & 0 & -1
\end{array}\right)
$$

The rotation matrix has a block-diagonal form

$$
M(\eta, \phi)=\left(\begin{array}{cccc}
e^{\eta} & 0 & 0 & 0 \\
0 & e^{-\eta} & 0 & 0 \\
0 & 0 & \cos \phi & \sin \phi \\
0 & 0 & -\sin \phi & \cos \phi
\end{array}\right)
$$

so that $M^{-1}(\eta, \phi)=M(-\eta,-\phi)$.

We can define the wavelet transform in light-cone coordinates in the same way as in Euclidean space using the representation of the affine group

$$
x^{\prime}=a M(\eta, \phi) x+b,
$$

defined in $L^{1}$ norm as

$$
U(a, b, \eta, \phi) u(x)=\frac{1}{a^{4}} u\left(M^{-1}(\eta, \phi) \frac{x-b}{a}\right) .
$$

we have the definition of wavelet coefficients of a function $f(x)$ with respect to the basic wavelet $g$ as follows

$$
\begin{aligned}
W_{a, b, \eta, \phi}[f] & =\frac{\int d x_{+} d x_{-} d^{2} \mathbf{x}_{\perp} \frac{1}{a^{4}} \times}{g\left(M^{-1}(\eta, \phi) \frac{x-b}{a}\right)} f\left(x_{+}, x_{-}, \mathbf{x}_{\perp}\right) .
\end{aligned}
$$

In contrast to wavelet transform in Euclidean space, where the basic wavelet $g$ can be defined globally on $\mathbb{R}^{d}$, the basic wavelet in Minkowski space is to be defined separately in four domains impassible by Lorentz rotations:

$$
\begin{aligned}
& A_{1}: k_{+}>0, k_{-}<0 ; A_{2}: k_{+}<0, k_{-}>0 \\
& A_{3}: k_{+}>0, k_{-}>0 ; A_{4}: k_{+}<0, k_{-}<0
\end{aligned}
$$

where $k$ is wave vector, $k_{ \pm}=\frac{\omega \pm k_{z}}{\sqrt{2}}$. Whence we have four separate wavelets in these four domains [35]:

$$
g_{i}(x)=\int_{A_{i}} e^{\imath k x} \tilde{g}(k) \frac{d^{4} k}{(2 \pi)^{4}} .
$$

We assert the following definition of the Fourier transform in light cone coordinates:

$$
\begin{array}{r}
f\left(x_{+}, x_{-}, \mathbf{x}_{\perp}\right)=\int e^{\imath k_{-} x_{+}+\imath k_{+} x_{-}-\imath \mathbf{k}_{\perp} \mathbf{x}_{\perp}} \tilde{f}\left(k_{-}, k_{+}, \mathbf{k}_{\perp}\right) \times \\
\frac{d k_{+} d k_{-} d^{2} \mathbf{k}_{\perp}}{(2 \pi)^{4}} .
\end{array}
$$

Substituting the Fourier images into the definition (22) we get

$$
\begin{gathered}
W_{a b \eta \phi}^{i}=\int_{A_{i}} e^{\imath k_{-} b_{+}+\imath k_{+} b_{-}-\imath \mathbf{k}_{\perp} \mathbf{b}_{\perp}} \tilde{f}\left(k_{-}, k_{+}, \mathbf{k}_{\perp}\right) \\
\overline{\tilde{g}}\left(a e^{\eta} k_{-}, a e^{-\eta} k_{+}, a R^{-1}(\phi) \mathbf{k}_{\perp}\right) \frac{d k_{+} d k_{-} d^{2} \mathbf{k}_{\perp}}{(2 \pi)^{4}} .
\end{gathered}
$$

In Fourier space the relation between Fourier coefficients and wavelet coefficients is therefore the same as in $\mathbb{R}^{d}$ :

$$
\tilde{W}_{a \eta \phi}(k)=\tilde{f}(k) \overline{\tilde{g}}\left(a M^{-1}(\eta, \phi) k\right) .
$$

Similarly, the reconstruction formula is [36]: 


$$
\begin{aligned}
f(x) & =\sum_{i=1}^{4} \frac{1}{C_{g_{i}}} \int_{-\infty}^{\infty} d \eta \int_{0}^{2 \pi} d \phi \int_{0}^{\infty} \frac{d a}{a} \int_{M_{1}^{4}} d b_{+} d b_{-} d^{2} \mathbf{b}_{\perp} \frac{1}{a^{4}} g_{i}\left(M^{-1}(\eta) \frac{\xi-b}{a}\right) W_{a b \eta \phi}^{i} \\
& =\sum_{i=1}^{4} \frac{1}{C_{g_{i}}} \int_{-\infty}^{\infty} d \eta \int_{0}^{2 \pi} d \phi \int_{0}^{\infty} \frac{d a}{a} \int_{A_{i}} \frac{d k_{+} d k_{-} d^{2} \mathbf{k}_{\perp}}{(2 \pi)^{4}} e^{\imath k_{-} x_{+}+\imath k_{+} x_{-}-\imath \mathbf{k}_{\perp} \mathbf{x}_{\perp}} \tilde{W}_{a \eta \phi}(k) \tilde{g}\left(a k_{-} e^{\eta}, a k_{+} e^{-\eta}, a R^{-1}(\phi) \mathbf{k}_{\perp}\right)
\end{aligned}
$$

If the problem is axially symmetric the azimuthal part of integration $(\phi)$ can be dropped. It is also convenient to use logarithmic scale $a=e^{u}$ to study different rapidity domains.

\section{E. Choice of the basic wavelet}

The choice of the basis of wavelet decomposition is always a subtle question, specially in quantum field theory. (The best choice, as it was already emphasized in [1], would be the apparatus function of a classical measuring device interacting with quantum system.) Some basis is always tacitly assumed. Even describing the massless photons, which are not localized anywhere, by plane waves, the possibility of photon registration by photomultiplier implies its interaction with electron, and hence some scale and some localization.

If the continuous wavelet transform is used in place of the Fourier transform the choice of the basic function is constrained by the feasibility of the analytical integration in Feynman diagrams on one hand, and by the possibility to understand this basic function as a localized (quasi) particle. The latter being claimed by some authors to be important for Minkowski space [37], seems questionable for Euclidean space calculations. If the wavelet transform is performed on a lattice there is a bias that only the similarity properties are important, rather than the shape of wavelet $[12,14]$. The question whether or not the basic wavelet should satisfy some equation of motion is still not clear. We are also not aware of the effect of the discrete symmetries of the basic wavelet.

To justify our choice of the derivatives of the Gaussian as the basic wavelets, we present the following heuristic consideration, inferred from the coherent states theory [33]. Let us introduce a localized wave packet in Fourier space

$$
\tilde{g}(t, k)=e^{-\imath t k-k^{2} / 2} .
$$

If the wave packet is considered in Minkowski space, then $k^{2}=0$ can be assumed for the photon and the whole equation (25) turns to be a plane wave. Otherwise it is a localized wave. If $t$ is time the packet (25) is a gaussian wave packet at initial time $t=0$. At finite but small instants of time the wavepacket can be approximated by its Taylor expansion

$$
\tilde{g}(t, k)=\tilde{g}_{0}(k)+\frac{t}{1 !} \tilde{g}_{1}(k)+\frac{t^{2}}{2 !} \tilde{g}_{2}(k)+O\left(t^{3}\right),
$$

where the expansion coefficients

$$
\tilde{g}_{n}(k)=\left.\frac{d^{n}}{d t^{n}} \tilde{g}(t, k)\right|_{t=0}
$$

are responsible for the shape of the packet at the time scales at which 1, 2 or $n$ excitations are significant. Clearly $g_{n}(x)$ are the excitations of the harmonic oscillator, with $g_{1}$ being the first excitation, see Fig. 5 .

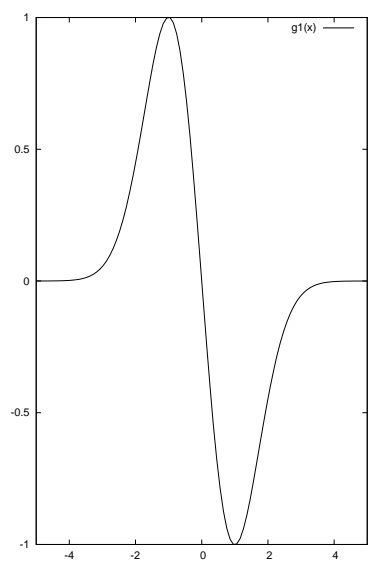

FIG. 5: Graph of $g_{1}$ wavelet: $g_{1}(x)=-x e^{-x^{2} / 2}$

\section{GAUGE THEORIES}

\section{A. QED}

Quantum electrodynamics is the simplest case of gauge theory. The local $U(1)$ invariance of the fermion field

$$
\psi(x) \rightarrow e^{-\imath e \Lambda(x)} \psi(x)
$$

is accompanied by the gradient invariance of the vector field $A_{\mu}(x)$, the electromagnetic field,

$$
A_{\mu}(x) \rightarrow A_{\mu}(x)+\partial_{\mu} \Lambda(x)
$$

to keep the total action $S(\bar{\psi}, \psi, A)=\int L d^{4} x$ invariant under the local $U(1)$ transform generated by $\Lambda(x)$. The interaction of the charged fermion field $\psi$ with electromagnetic field $A_{\mu}$ is introduced by substitution of ordinary derivatives $\partial_{\mu}$ to covariant derivatives

$$
D_{\mu}=\partial_{\mu}+\imath e A_{\mu}(x),
$$


with $e$ being the charge of the fermion.

The Lagrangian of QED has the (Euclidean) form

$$
L=\bar{\psi}(x)(\not D+\imath m) \psi(x)+\frac{1}{4} F_{\mu \nu} F^{\mu \nu}+\underbrace{\frac{1}{2 \alpha}\left(\partial_{\mu} A_{\mu}\right)^{2}}_{\text {gauge fixing }},
$$$$
\text { with } F_{\mu \nu}=\partial_{\mu} A_{\nu}-\partial_{\nu} A_{\mu}
$$

being the field strength tensor of the electromagnetic field $A$, and $\gamma_{\mu}$ being the Dirac $\gamma$-matrices.

The straightforward application of the Feynman integral to the gauge theory with the Lagrangian (27) would be inefficient for the integration over the field $A(x)$ would contain an infinite set of physically equivalent field configurations. For this purpose the gauge fixing, which restricts the integration only to gauge-nonequivalent field configurations was introduced by Faddeev and Popov [38].

Quantum electrodynamics is the most firmly established and most verified field theory model in physics of elementary particles. The probability amplitude of scattering obtained at a tree-level are in fairly good agreement with classical scattering theory. Starting from one loop level the Feynman integrals are formally divergent, and the physical results are derived using the renormalization invariance of QED. The most accurate tests for the renormalized calculations of the electron-photon interaction are the Lamb shift of the Hydrogen-like ion energy levels and the anomalous magnetic momentum of the electron [39-42].

In one-loop approximation the radiation corrections in QED come from three primitive Feynman graphs: fermion self-energy $\Sigma(p)$, vacuum polarization operator $\Pi_{\mu \nu}(p)$, and the vertex function $\Gamma_{\rho}(p, q)$. In Euclidean space the equations for the above three graphs have the form:

Electron self-energy

$$
\Sigma(p)=-e^{2} \int \frac{d^{4} q}{(2 \pi)^{4}} \gamma_{\mu} \frac{-\imath}{\not p-\not p+m} \gamma_{\nu} \frac{\delta_{\mu \nu}}{q^{2}}
$$

gives the corrections to the bare electron mass $m_{0}$ related to irradiation of virtual photons.

Vacuum polarization diagram

$$
\Pi_{\mu \nu}(p)=-e^{2} \int \frac{d^{4} q}{(2 \pi)^{4}} \operatorname{Sp}\left[\gamma_{\mu} \frac{1}{\not p+\not p+m} \gamma_{\nu} \frac{1}{\not q+m}\right]
$$

could be expected to give the nonzero corrections to the photon mass, but due to gauge invariance the photon mass remains zero, instead the one loop contribution (29) renormalizes the electron charge at small distances, therefore modifies the Coulomb interaction by screening the bare electron charge $e_{0}$ by virtual electron-positron pairs polarizing the vacuum at small distances. This diagram contributes to the Lamb shift of the atom energy levels.
One-loop vertex function

$\Gamma_{\rho}(p, q)=-\imath e^{3} \int \frac{d^{4} f}{(2 \pi)^{4}} \gamma_{\tau} \frac{1}{\not p+f+m} \gamma_{\rho} \frac{1}{f+\not d+m} \gamma_{\sigma} \frac{\delta_{\tau \sigma}}{f^{2}}$

determines the anomalous magnetic moment of the electron.

All three integrals $(28,29,30)$ are divergent. Their evaluation involves regularization procedures. The most common is the dimensional regularization with all integrals taken in formal $d=2 \omega$ dimension with physical value $\omega=2$. In this way the divergences come as poles in $\epsilon=2-\omega$, see e.g. [23, 25, 43].

\section{B. One loop corrections in wavelet-based theory}

The evaluation of Feynman diagrams with fermions and gauge fields in wavelet-based Euclidean theory is similar to that of scalar theory (15). The evaluation of the one-loop radiative corrections for the scale-dependent fields give finite results by construction with no regularization procedure required.

a. Electron self-energy. For the scale components of the electron self-energy diagram, we get

$\frac{\Sigma^{(A)}(p)}{\tilde{g}(a p) \tilde{g}\left(-a^{\prime} p\right)}=-\imath e^{2} \int \frac{d^{4} q}{(2 \pi)^{4}} \frac{F_{A}(p, q) \gamma_{\mu}\left[\frac{\not p}{2}-\not p-m\right] \gamma_{\mu}}{\left[\left(\frac{p}{2}-q\right)^{2}+m^{2}\right]\left[\frac{p}{2}+q\right]^{2}}$,

where $A$ is the minimal scale of two external lines shown in Fig. 6: $A=\min \left(a, a^{\prime}\right)$. The regularizing function $F_{A}(p, q)$ is the result of integration over the scales of two internal lines. For the isotropic basic wavelet $g$ the regu-

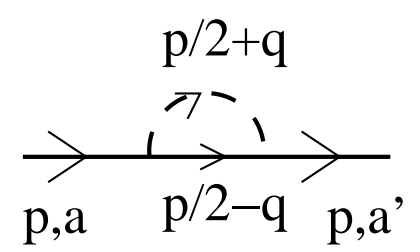

FIG. 6: Electron self-energy diagram

larizing function is given by (14):

$$
F_{A}(p, q)=f^{2}(A(p / 2-q)) f^{2}(A(p / 2+q)) .
$$

Introducing the dimensionless variable $\mathbf{y}=\mathbf{q} /|\mathbf{p}|$, after straightforward algebra, we can perform the integration in Euclidean space:

$$
\begin{gathered}
\frac{\Sigma^{(A)}(p)}{\tilde{g}(a p) \tilde{g}\left(-a^{\prime} p\right)}=-\imath e^{2} \int \frac{d^{4} y}{(2 \pi)^{4}} F_{A}(p,|p| y) \times \\
\times \frac{\not p+4 m-2|p| \not y}{\left[y^{2}+\frac{1}{4}-y \cos \theta-\frac{m^{2}}{p^{2}}\right]\left[y^{2}+\frac{1}{4}+y \cos \theta\right]} .
\end{gathered}
$$


where $\theta$ is the Euclidean angle between the $p$ and the $q$ directions. In high energy limit, $p^{2} \gg 4 m^{2}$, the contribution of last term in the numerator of (33) vanishes for the symmetry, and the equation (33) can be easily integrated in angle variable (C1):

$$
\begin{array}{rr}
\frac{\Sigma^{(A)}(p)}{\tilde{g}(a p) \tilde{g}\left(-a^{\prime} p\right)}= & -\frac{\imath e^{2}}{4 \pi^{2}} R_{1}(p)(\not p+4 m) \quad \text { where: } \\
R_{1}(p)=\int_{0}^{\infty} d y y F_{A}(p,|p| y)\left[1-\sqrt{1-\frac{1}{\beta^{2}(y)}}\right], \\
\beta(y)=y+\frac{1}{4 y} .
\end{array}
$$

The integral $R_{1}(p)$ is finite for any wavelet cutoff function (14). For the $g_{1}$ wavelet we get

$$
R_{1}(p)=e^{-A^{2} p^{2}} \int_{0}^{\infty} d y y e^{-4 A^{2} p^{2} y^{2}}\left[1-\sqrt{1-\frac{1}{\beta^{2}}}\right] .
$$

After a simple algebra this gives

$$
\begin{aligned}
R_{1}(p) & =\frac{1}{8 A^{2} p^{2}}\left(2 A^{2} p^{2} \operatorname{Ei}_{1}\left(A^{2} p^{2}\right)-4 A^{2} p^{2} \operatorname{Ei}_{1}\left(2 A^{2} p^{2}\right)\right. \\
& - \\
& \left.e^{-A^{2} p^{2}}+2 e^{-2 A^{2} p^{2}}\right)
\end{aligned}
$$

b. Vacuum polarization diagram The vacuum polarization diagram in quantum electrodynamics of scaledependent fields is obtained by integration over the scale variables of the fermion loop shown in Fig. 7:

$$
\begin{array}{rlr}
\frac{\Pi_{\mu \nu}^{(A)}(p)}{\tilde{g}(a p) \tilde{g}\left(-a^{\prime} p\right)} & = & -e^{2} \int \frac{d^{4} q}{(2 \pi)^{4}} F_{A}(p, q) \times \\
& \times & \frac{\operatorname{Sp}\left(\gamma_{\mu}(\not q+\not p / 2-m) \gamma_{\nu}(\not t-\not p / 2-m)\right)}{\left[(q+p / 2)^{2}+m^{2}\right]\left[(q-p / 2)^{2}+m^{2}\right]} \\
& = & -4 e^{2} \int \frac{d^{4} q}{(2 \pi)^{4}} F_{A}(p, q) \times \\
& \times & \frac{2 q_{\mu} q_{\nu}-\frac{1}{2} p_{\mu} p_{\nu}+\delta_{\mu \nu}\left(\frac{p^{2}}{4}-q^{2}-m^{2}\right)}{\left[\left(q+\frac{p}{2}\right)^{2}+m^{2}\right]\left[\left(q-\frac{p}{2}\right)^{2}+m^{2}\right]} .
\end{array}
$$

Similarly to previous diagram we use the dimensionless

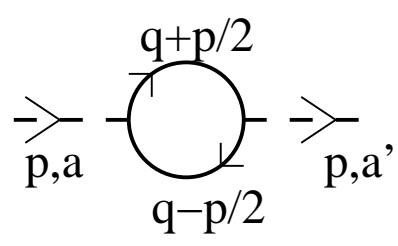

FIG. 7: Vacuum polarization diagram in (Euclidean) scaledependent QED

variable $y$ and integrate over the angle variable. The momentum integration in equation (35) is straightforward: having expressed all momenta in units of electron mass $m$, we express the loop momentum in terms of the photon momentum and perform the integration over the angle variable:

$$
\begin{aligned}
& \frac{\Pi_{\mu \nu}^{(A)}}{\tilde{g}(a p) \tilde{g}\left(-a^{\prime} p\right)}=-\frac{e^{2}}{\pi^{3}}\left(m^{2} p^{2}\right) \int_{0}^{\infty} d y y F_{A}(m p, m p y) \times \\
& \times \int_{0}^{\pi} d \theta \sin ^{2} \theta \frac{2 y_{\mu} y_{\nu}-\frac{1}{2} \frac{p_{\mu} p_{\nu}}{p^{2}}+\delta_{\mu \nu}\left(\frac{1}{4}-y^{2}-\frac{1}{p^{2}}\right)}{\left[\frac{\frac{1}{4}+y^{2}+\frac{1}{p^{2}}}{y}+\cos \theta\right]\left[\frac{\frac{1}{4}+y^{2}+\frac{1}{p^{2}}}{y}-\cos \theta\right]}
\end{aligned}
$$

where $p$ is dimensionless, i.e. is expressed in units of $m$. Introducing the notation $\beta(y) \equiv \frac{\frac{1}{4}+y^{2}+\frac{1}{p^{2}}}{y}$ and using the substitution $y_{\mu} y_{\nu} \rightarrow A y^{2} \delta_{\mu \nu}+B y^{2} \frac{p_{\mu} p_{\nu}}{p^{2}}$, we get

$$
\begin{array}{r}
\frac{\Pi_{\mu \nu}^{(A)}}{\tilde{g}(a p) \tilde{g}\left(-a^{\prime} p\right)}=-\frac{e^{2}}{\pi^{3}}\left(m^{2} p^{2}\right) \int_{0}^{\infty} d y y F_{A}(m p, m p y) \times \\
\times \int_{0}^{\pi} d \theta \sin ^{2} \theta \frac{\delta_{\mu \nu}\left((2 A-1) y^{2}+\frac{1}{4}-\frac{1}{p^{2}}\right)+\frac{p_{\mu} p_{\nu}}{p^{2}}\left(2 B y^{2}-\frac{1}{2}\right)}{\beta^{2}(y)-\cos ^{2} \theta}
\end{array}
$$

where $A$ and $B$ depend only on the modulus of $y$, but not on the direction, and can be expressed in terms of angle integrals (C1).

Finally, writing the polarization operator as a sum of transversal and longitudinal parts, we have the equations

$$
\begin{aligned}
& \frac{\Pi_{\mu \nu}^{(A)}(p)}{\tilde{g}(a p) \tilde{g}\left(-a^{\prime} p\right)} \equiv \quad \delta_{\mu \nu} \pi_{T}^{(A)}+\frac{p_{\mu} p_{\nu}}{p^{2}} \pi_{L}^{(A)} \\
& =\quad\left(\delta_{\mu \nu}-\frac{p_{\mu} p_{\nu}}{p^{2}}\right) \pi_{T}^{(A)}+X^{(A)} \frac{p_{\mu} p_{\nu}}{p^{2}}, \\
& \pi_{T}^{(A)}=\quad-\frac{e^{2}}{3 \pi^{2}} m^{2} p^{2} \int_{0}^{\infty} d y y F_{A}(m p, m p y)\left[y^{2}+\right. \\
& +\left(1-\sqrt{\frac{\frac{1}{16}+y^{4}+\frac{1}{p^{4}}-\frac{y^{2}}{2}+\frac{1}{2 p^{2}}+\frac{2 y^{2}}{p^{2}}}{\left(\frac{1}{4}+y^{2}+\frac{1}{p^{2}}\right)^{2}}}\right) \\
& \left.\times \quad\left(\frac{5}{8}-\frac{4}{p^{2}}-\frac{2}{p^{4}}-2 y^{2}\left(1+\frac{2}{p^{2}}\right)-2 y^{4}\right)\right] \\
& \pi_{L}^{(A)}=-\frac{e^{2}}{3 \pi^{2}} m^{2} p^{2} \int_{0}^{\infty} d y y F_{A}(m p, m p y)\left[-4 y^{2}+\right. \\
& +\left(1-\sqrt{\frac{\frac{1}{16}+y^{4}+\frac{1}{p^{4}}-\frac{y^{2}}{2}+\frac{1}{2 p^{2}}+\frac{2 y^{2}}{p^{2}}}{\left(\frac{1}{4}+y^{2}+\frac{1}{p^{2}}\right)^{2}}}\right) \\
& \left.\times \quad\left(8 y^{4}+2 y^{2}\left(1+\frac{8}{p^{2}}\right)+\frac{4}{p^{2}}+\frac{8}{p^{4}}-1\right)\right],
\end{aligned}
$$


where

$$
\begin{array}{rlrl}
X^{(A)} & = & \pi_{L}^{(A)}+\pi_{T}^{(A)}=\frac{e^{2} m^{2} p^{2}}{\pi^{2}} \int_{0}^{\infty} d y y F_{A}(m p, m p y) \\
\times & {\left[y^{2}-\left(1-\sqrt{\frac{\frac{1}{16}+y^{4}+\frac{1}{p^{4}}-\frac{y^{2}}{2}+\frac{1}{2 p^{2}}+\frac{2 y^{2}}{p^{2}}}{\left(\frac{1}{4}+y^{2}+\frac{1}{p^{2}}\right)^{2}}}\right)\right.} \\
\times & \left.\left(2 y^{4}+4 \frac{y^{2}}{p^{2}}+\frac{2}{p^{4}}-\frac{1}{8}\right)\right] .
\end{array}
$$

The integrals above are finite and can be easily evaluated in large momenta limit, $p^{2} \gg 4$, introducing the dimensionless scale $a=A m$.

As an example we can evaluate the vacuum polarization operator for $g_{1}$ wavelet. For $g_{1}$ wavelet the regularizing function

$$
F_{A}(p, q)=\exp \left(-A^{2} p^{2}-4 A^{2} q^{2}\right) .
$$

Hence for large $p^{2} \gg 4$ the integral (36) can be evaluated by substitution $y^{2}=t[20]$ :

$$
\begin{aligned}
\pi_{T}^{(A)} & =\quad-\frac{e^{2}}{6 \pi^{2}} m^{2} p^{2}\left\{\frac{e^{-a^{2} p^{2}}}{8 a^{6} p^{6}}\left(4 a^{4} p^{4}-a^{2} p^{2}-1\right)+\frac{e^{-2 a^{2} p^{2}}}{8 a^{6} p^{6}}\right. \\
& \left.\times \quad\left(1-4 a^{4} p^{4}+2 a^{2} p^{2}\right)-\frac{1}{2} \operatorname{Ei}_{1}\left(a^{2} p^{2}\right)+\operatorname{Ei}_{1}\left(2 a^{2} p^{2}\right)\right\} .
\end{aligned}
$$

Similarly, the longtitudinal term $X^{A}$ evaluated with $g_{1}$ wavelet in the limit $p^{2} \gg 4$ is equal to

$$
X^{A}=\frac{e^{2} m^{2} p^{2}}{16 \pi^{2}} \frac{e^{-a^{2} p^{2}}\left(a^{2} p^{2}-1+e^{-a^{2} p^{2}}\right)}{a^{6} p^{6}}
$$

In the limit of small scales $a p \ll 1$ the equation (37) does not depend on $p: X^{A} \propto \frac{1}{a^{2}}$. Therefore the whole equation (36) is similar to the result obtained by PauliVillars regularization of the vacuum polarization

$$
\Pi_{\mu \nu}^{M}(p)=c M^{2} \delta_{\mu \nu}+\left(p^{2} \delta_{\mu \nu}-p_{\mu} p_{\nu}\right) F\left(\frac{p^{2}}{4 m^{2}}, \frac{m}{M}\right),
$$

where $M \rightarrow \infty$ is a regularizing mass [43]. The gauge invariance is restored if the multiscale diagram (35) is integrated over all scales. In this limit the theory can be subjected to dimensional regularization [1].

c. Vertex function. The one-loop contribution to the QED vertex function for the theory with scaledependent matter fields is shown in Fig. 8 below. The equation, which corresponds to the vertex diagram Fig. (8) can be casted in the form

$$
\begin{array}{r}
-\imath e \frac{\Gamma_{\mu, r}^{(A)}}{\tilde{g}\left(-p a^{\prime}\right) \tilde{g}(-q r) \tilde{g}(k a)}=(-\imath e)^{3} \int \frac{d^{4} l}{(2 \pi)^{4}} \gamma_{\alpha} G(p-f) \gamma_{\mu} \times \\
\times G(k-f) \gamma_{\beta} D_{\alpha \beta} F_{A}(p-f) F_{A}(k-f) F_{A}(f) .
\end{array}
$$

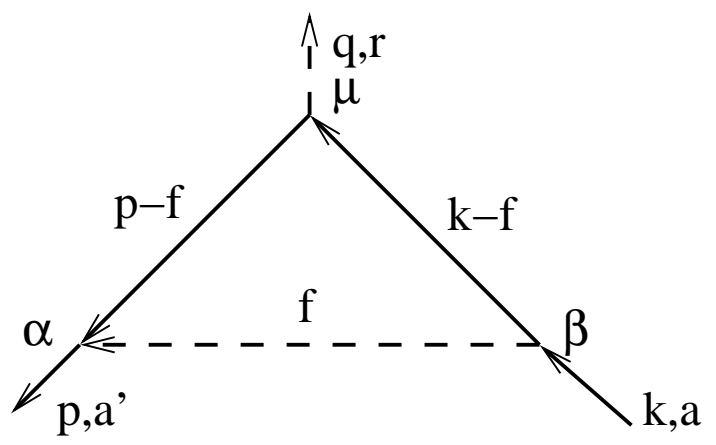

FIG. 8: One-loop vertex function in scale-dependent QED

The explicit substitution with photon propagator taken in Feynman gauge gives

$$
\begin{array}{r}
\imath e \frac{\Gamma_{\mu, r}^{(A)}}{\tilde{g}\left(-p a^{\prime}\right) \tilde{g}(-q r) \tilde{g}(k a)}=(-\imath e)^{3} \int \frac{d^{4} f}{(2 \pi)^{4}} \gamma_{\alpha} \frac{\not p-f-m}{(p-f)^{2}+m^{2}} \\
\times \gamma_{\mu} \frac{\not k-f-m}{(k-f)^{2}+m^{2}} \gamma_{\alpha} \frac{1}{f^{2}} F_{A}(p-f) F_{A}(k-f) F_{A}(f)
\end{array}
$$

Representing the numerator of the latter equation in the form

$$
\begin{array}{cr}
A_{\mu}= & \gamma_{\alpha}(\not p-f) \gamma_{\mu}(\not k-f) \gamma_{\alpha}- \\
- & m\left[(\not p-f) \gamma_{\mu}+\gamma_{\mu}(\not k-f)\right]+2 m^{2} \gamma_{\mu},
\end{array}
$$

it can be seen that the right-hand side of the equation (38) can be represented as a linear combination of three finite integrals $\left(J^{(0)}, J_{\mu}^{(1)}, J_{\mu \nu}^{(2)}\right)$ presented in Appendix C, in analog to their divergent counterparts in Minkowski space [44]. After some algebra the vertex (38) turns to be

$$
\begin{array}{rr}
\frac{\Gamma_{\mu, r}^{(A)}}{\tilde{g}\left(-p a^{\prime}\right) \tilde{g}(-q r) \tilde{g}(k a)} & =e^{2} \gamma_{\alpha}\left[\left(\not p \gamma_{\mu} \not k-m \not p \gamma_{\mu}-m \gamma_{\mu} \not k\right) J^{(0)}\right. \\
- & \left(\gamma_{\nu} \gamma_{\mu} \not k+\not p \gamma_{\mu} \gamma_{\nu}+2 m \delta_{\mu \nu}\right) J_{\nu}^{(1)} \\
+ & \left.\gamma_{\lambda} \gamma_{\mu} \gamma_{\nu} J_{\lambda \nu}^{(2)}\right] \gamma_{\alpha} .
\end{array}
$$

\section{Ward-Takahashi identities}

The Ward-Takahashi identity in spinor electrodynamics relates the vertex function to the difference of fermion propagators:

$$
q_{\mu} \Gamma_{\mu}(p, q, p+q)=G^{-1}(p+q)-G^{-1}(p),
$$

where $G(p)$ is the complete fermion propagator. The identity (40) is a helpful constraint which ensures the gauge invariance of the renormalized QED in any order of perturbation theory $[45,46]$. The constraint (40) makes 
the perturbation expansion gauge invariant at the presence of the gauge fixing terms in the QED generating functional.

In wavelet-based theory, where the fields explicitly depend on scale the divergence do not appear in the Feynman diagrams, but the evaluation of integrals in internal lines with the integration scales constrained by the minimal scale of external lines may spoil the gauge invariance of the complete propagator. To prevent this the WardTakahashi identities are required.

At the absence of gauge-fixing terms in the Lagrangian (27), the generating functional

$$
e^{-Z[J, \bar{\chi}, \chi]}=\int \mathcal{D} A \mathcal{D} \bar{\psi} \mathcal{D} \psi e^{-\int d^{4} x(L(\psi, \bar{\psi}, A)+J A+\imath \bar{\chi} \psi+\imath \bar{\psi} \chi)},
$$

with $L(\psi, \bar{\psi}, A)$ given by the equation (27), would be invariant under the gauge transformations (26) if no source term $-\int d^{4} x\left(J_{\mu} A_{\mu}+\imath \bar{\chi} \psi+\imath \bar{\psi} \chi\right)$ is present.

In the framework of scale-dependent functions the gauge field $A_{\mu}(x)$ is expressed in terms of its wavelet coefficients $A_{\mu a}(b)$ :

$$
A_{\mu}(x)=\frac{1}{C_{g}} \int_{\mathbb{R}_{+} \times \mathbb{R}^{d}} \frac{1}{a^{d}} g\left(\frac{x-b}{a}\right) A_{\mu a}(b) \frac{d a d^{d} b}{a}
$$

(with the angular part of wavelet transform (5) dropped for simplicity). In view of linearity of wavelet transform we may infer the gauge transform of the scale components to have the form

$$
A_{\mu a}^{\prime}(x)=A_{\mu a}(x)+\frac{\partial \Lambda_{a}(x)}{\partial x_{\mu}},
$$

where

$$
\Lambda_{a}(x)=\int_{\mathbb{R}^{d}} \frac{1}{a^{d}} \bar{g}\left(\frac{y-x}{a}\right) \Lambda(y) d^{d} y
$$

is the scale component of the gauge function (26). That is the gauge transform of the abelian gauge field $A_{\mu a}(x)$ is a projection of the (no-scale) gauge field $A_{\mu}(x)$ onto the space of resolution $a$.

Since the free Lagrangian of QED is gauge-invariant by construction, the derivative of the Ward-Takahashi identities turns into evaluation of the functional overage of the variation of source and gauge fixing terms under inifinitesimal gauge transform

$$
\delta A_{\mu}=\partial_{\mu} \Lambda, \quad \delta \psi=-\imath e \Lambda \psi, \quad \delta \bar{\psi}=\imath e \Lambda \bar{\psi},
$$

where $\Lambda=\Lambda(x)$ is considered to be small. Under this variation the integrand in the functional integral (41), after integration by parts, acquires a multiplicative factor $e^{\delta_{\Lambda}}$, with

$$
\delta_{\Lambda} \equiv \int d^{d} x\left[-\frac{1}{\alpha} \partial^{2}\left(\partial_{\mu} A_{\mu}\right)+\partial_{\mu} J_{\mu}+e(\bar{\psi} \chi-\bar{\chi} \psi)\right] \Lambda(x) .
$$

Considering $\delta_{\Lambda}$ as small we can approximate $e^{\delta_{\Lambda}} \approx 1+\delta_{\Lambda}$ and proceed with the derivation procedure from

$$
\delta=\left\langle\delta_{\Lambda}\right\rangle=0
$$

The standard procedure of the variation of action with a gauge fixing term [47] with respect to $\Lambda_{a}(x)$ (43) leads to the equations [48]:

$$
\begin{array}{r}
q_{\mu} \Gamma_{\mu a_{4} a_{3} a_{1}}(p, q, p+q)=\quad \int \frac{d a_{2}}{a_{2}} G_{a_{1} a_{2}}^{-1}(p+q) \tilde{M}_{a_{2} a_{3} a_{4}}(p+q, q, p)-\int \frac{d a_{2}}{a_{2}} \tilde{M}_{a_{1} a_{3} a_{2}}(p+q, q, p) G_{a_{2} a_{4}}^{-1}(p), \\
\text { where } \\
\tilde{M}_{a_{1} a_{2} a_{3}}\left(k_{1}, k_{2}, k_{3}\right)=(2 \pi)^{d} \delta^{d}\left(k_{1}-k_{2}-k_{3}\right) \overline{\tilde{g}}\left(a_{1} k_{1}\right) \tilde{g}\left(a_{2} k_{2}\right) \tilde{g}\left(a_{3} k_{3}\right) .
\end{array}
$$

The equation (44) is exactly the wavelet transform of the standard Ward-Takahashi identity (40).

\section{QCD example}

Same as in QED we can evaluate the gluon vacuum polarization operator using $g_{1}$ as the basic wavelet. The corresponding one-loop diagram is shown in Diagram 45.

$$
=-\frac{g^{2}}{2} f^{A B C} f^{B D C} \int \frac{d^{4} l}{(2 \pi)^{4}} \frac{N_{\mu \nu}(l, p) F_{\mathcal{A}}(l+p, l)}{l^{2}(l+p)^{2}},
$$

where

$$
\begin{array}{rrr}
N_{\mu \nu}(l, p)= & 10 l_{\mu} l_{\nu}+5\left(l_{\mu} p_{\nu}+l_{\nu} p_{\mu}\right)-2 p_{\mu} p_{\nu} \\
+ & & (p-l)^{2} \delta_{\mu \nu}+(2 p+l)^{2} \delta_{\mu \nu}
\end{array}
$$

is the tensor structure of the vacuum polarization diagram (45) in $\mathbb{R}^{4}$ Euclidean space. $\mathcal{A}$ is the minimal scale 
of two external lines. The regularizing function, if calculated with $g_{1}$ wavelet, has the form (32):

$$
F_{\mathcal{A}}(l+p, l)=\exp \left(-2 \mathcal{A}^{2}(l+p)^{2}-2 \mathcal{A}^{2} l^{2}\right) .
$$

Symmetrizing the loop momenta in equation (45) by substitution $l=q-\frac{p}{2}$, we obtain

$$
\begin{array}{r}
\Pi_{A B, \mu \nu}^{(\mathcal{A})}(p)=-\frac{g^{2}}{2} f^{A C D} f^{B D C} \int \frac{d^{4} q}{(2 \pi)^{4}} F_{\mathcal{A}}(p, q) \times \\
\frac{10 q_{\mu} q_{\nu}-\frac{9}{2} p_{\mu} p_{\nu}+\delta_{\mu \nu}\left(\frac{9}{2} p^{2}+2 q^{2}\right)}{\left[q^{2}-\frac{p^{2}}{4}\right]^{2}}
\end{array}
$$

For $g_{1}$ wavelet the regularizing function $F_{\mathcal{A}}(p, q)$ is given by equation (32).
The integral (46) can be easily evaluated in infrared limit where ordinary QCD is divergent:

$$
\Pi_{A B, \mu \nu}^{\left(\mathcal{A}, g_{1}\right)}(p \rightarrow 0)=-g^{2} f^{A C D} f^{B D C} \int \frac{d^{4} q}{(2 \pi)^{4}} \frac{e^{-4 \mathcal{A}^{2} q^{2}}}{q^{4}}\left[5 q_{\mu} q_{\nu}+q^{2} \delta_{\mu \nu}\right]
$$

Making use of the isotropy

$$
d^{4} q \rightarrow 2 \pi^{2} q^{3} d q, \quad q_{\mu} q_{\nu} \rightarrow \delta_{\mu \nu} \frac{q^{2}}{4}
$$

we get

$$
\begin{array}{r}
\Pi_{A B, \mu \nu}^{\left(\mathcal{A}, g_{1}\right)}(p \rightarrow 0)=-\frac{9 g^{2} f^{A C D} f^{B D C} \delta_{\mu \nu}}{32} \int_{0}^{\infty} q d q e^{-4 \mathcal{A}^{2} q^{2}} \\
=-\frac{9 g^{2} f^{A C D} f^{B D C} \delta_{\mu \nu}}{256 \mathcal{A}^{2}} .
\end{array}
$$

Similar contribution comes from the ghost loop.

\section{CONCLUSION}

In this paper we developed a regularization method for quantum field theory based on a continuous wavelet transform. Regardless significant amount of works devoted to wavelet-based regularization in different quantum field theory models $[11,12,49]$, all those are basically the lattice theories. The novelty of the present approach, developed by the authors [1, 13, 50], consists in using continuous wavelet transform to substitute the local fields $\phi(x)$ by the scale-dependent fields $\phi_{a}(x)$, defined as wavelet-coefficients of the physical field. Substitution of such fields into the action, supplied by appropriate causality assumptions and operator ordering $[1,2,28]$, results in effective regularization of Feynman graphs, which makes each internal line decay as an effective factor $\propto e^{-p^{2} A^{2}}$, where $A$ is the minimal scale of all internal lines, and $p$ is momentum.

Regulazation factors, that are technically similar to our approach, were already known in QCD. They are related to the modification of the gluon vacuum state to the instanton vacuum, with the parameter $A$ understood as the size of the instanton $[51,52]$. The difference between the instanton vacuum model and our model is that the scattered quark fields are local fields in the instanton model and only the interaction with instanton vacuum is smeared. In our approach the incident particles are nonlocal wave packets and only the integration over all scales makes the theory local.

The physics of using scale-dependent fields $\phi_{a}(x)$ instead of local fields $\phi(x)$ lies in the fact, that no physical quantity can be measured in a point, but in a region of nonzero size $a>0$. Thus only the finite resolution projections $\phi_{a}(x)$ of a quantum field $\phi$ are physically meaningful. The $n$-point Green functions for such fields constructed by our method are finite by construction and do not require regularization. The gauge invariance of the theory results in appropriate Ward-Takahashi identities, which are the projections of ordinary Ward-Takahashi identities onto finite resolution spaces.

The practical applications of our approach can be found in such physical settings where the separation of the field to the components of different scales is physically meaningful. Such models have been presented in QED calculations of the dependence of the Casimir force on the size of displacement in measurement [50], and also in application of quantum field theory methods to the calculation of correlations of the turbulent velocity fluctuations of different scales [53]. We strongly hope that, regardless the yet unsolved problem of deriving renormalization group equation in continuous limit of waveletbased theory, this method can be also applied for QCD calculations, where it was originally proposed [11]. 


\section{Acknowledgments}

The research was supported in part by RFBR Project 13-07-00409 and by the Program of Creation and Development of the National University of Science and Technology "MISiS". The authors are thankful to A.E.Dorokhov, V.G.Kadyshevsky, D.I.Kazakov and O.V.Teryaev for useful discussions, and to anonymous Referee for a number of valuable comments.

\section{Appendix A: Dirac $\gamma$-matrices in Euclidean space}

$$
\begin{array}{r}
\gamma_{\mu} \gamma_{\nu}+\gamma_{\nu} \gamma_{\mu}=-2 \delta_{\mu \nu} \\
\gamma_{\mu} \gamma_{\mu}=-4, \quad \gamma_{\mu} \not p \gamma_{\mu}=2 \not p
\end{array}
$$

Slashed vectors denote convolution with Dirac gammamatrices $\not k=\gamma_{\mu} k_{\mu}, \not k \not k=-k^{2}$

\section{Appendix B: Feynman rules in Euclidean space}

Photon propagator is taken in Feynman gauge:

$$
D(k)=\frac{\delta_{\mu \nu}}{k^{2}}
$$

Fermion propagator

$$
G_{E}^{(2)}(p)=\frac{-\imath}{\not p+m}=\imath \frac{\not p-m}{p^{2}+m^{2}}
$$

Electron-fermion vertex:

$$
-\imath e \gamma_{\rho}
$$

Besides that each fermion vertex results in extra sign - of the whole diagram.

\section{Appendix C: Functions and Integrals}

Exponential integral of the first type

$$
\operatorname{Ei}_{1}(z)=\int_{1}^{\infty} \frac{e^{-x z}}{x} d x
$$

Integrals for angle integration in Euclidean Green functions [20]:

$$
\begin{array}{lrl}
I_{k}(y) & \equiv & \int_{0}^{\pi} d \theta \frac{\sin ^{2} \theta \cos ^{2 k} \theta}{\beta^{2}(y)-\cos ^{2} \theta}, \\
I_{0}(y)= & \pi\left(1-\sqrt{1-\beta^{-2}(y)}\right), \\
I_{1}(y)= & -\frac{\pi}{2}+\beta^{2}(y) I_{0}(y),
\end{array}
$$

The constants $A, B$ for the vacuum polarization diagram (35) are given by $4 A+B=1, \quad A+B=I_{1} / I_{0}$, from where we get

$A=\frac{1}{3}+\frac{\pi}{6} I_{0}^{-1}(y)-\frac{1}{3} \beta^{2}(y), B=-\frac{1}{3}-\frac{2 \pi}{3} I_{0}^{-1}(y)+\frac{4}{3} \beta^{2}(y)$.

Integrals in one-loop fermion-photon vertex

$$
\begin{aligned}
J^{(0)} & =\int \frac{d^{4} f}{(2 \pi)^{4}} \frac{F_{A}(p-f) F_{A}(k-f) F_{A}(f)}{\left[(p-f)^{2}+m^{2}\right]\left[(k-f)^{2}+m^{2}\right] f^{2}} \\
J_{\mu}^{(1)} & =\int \frac{d^{4} f}{(2 \pi)^{4}} \frac{f_{\mu} F_{A}(p-f) F_{A}(k-f) F_{A}(f)}{\left[(p-f)^{2}+m^{2}\right]\left[(k-f)^{2}+m^{2}\right] f^{2}} \\
J_{\mu \nu}^{(2)} & =\int \frac{d^{4} f}{(2 \pi)^{4}} \frac{f_{\mu} f_{\nu} F_{A}(p-f) F_{A}(k-f) F_{A}(f)}{\left[(p-f)^{2}+m^{2}\right]\left[(k-f)^{2}+m^{2}\right] f^{2}}
\end{aligned}
$$

[1] M. V. Altaisky, Phys. Rev. D 81, 125003 (2010).

[2] J. D. Christensen and L. Crane, J. Math. Phys 46, 122502 (2005).

[3] J. C. Collins, Renormalization (Cambridge University Press, Cambridge, England, 1984).

[4] J. B. Kogut, Rev. Mod. Phys. 51, 659 (1979).

[5] V. Bornyakov, V. Mitrjushkin, and M. Muller-Preussker, Phys. Rev. D 81, 054503 (2010).

[6] L. P. Kadanoff, Physics 2, 263 (1966).

[7] K. Gawedzki and A. Kupiainen, Ann. Phys. 147, 198 (1983).

[8] J. Morlet, G. Arens, and I. Fourgeau, Geophysics 47, 203 (1982).

[9] C. Best and A. Schaefer (1994), URL arxiv.org: hep-lat/940212.

[10] I. G. Halliday and P. Suranyi, Nucl. Phys. B 436, 414 (1995).
[11] P. Federbush, Progr. Theor. Phys. 94, 1135 (1995).

[12] G. Battle, Wavelets and renormalization group (World Scientific, 1999).

[13] M. V. Altaisky, IOP Conf. Ser. 173, 893 (2003).

[14] F. Bulut and W. Polyzou (2013), URL arxiv.org: math-ph/1301.6570.

[15] A. L. Carey, Bull. Austr. Math. Soc. 15, 1 (1976).

[16] M. Duflo and C. C. Moore, J. Func. Anal. 21, 209 (1976).

[17] C. K. Chui, An Introduction to Wavelets (Academic Press Inc., 1992).

[18] C. R. Handy and R. Murenzi, Phys. Rev. A 54, 3754 (1996).

[19] E. Freysz, B. Pouligny, F. Argoul, and A. Arneodo, Phys. Rev. Lett. 64, 745 (1990).

[20] M. V. Altaisky, Symmetry, Integrability and Geometry: Methods and Applications 3, 105 (2007).

[21] D. Gitman and A. Shelepin, Eur. Phys. J. C 61, 111 
(2009).

[22] V. Varlamov, Int.J.Theor.Phys. 51, 1453 (2012).

[23] G. 't Hooft and M. Veltman, Nuclear Physics B 44, 189 (1972).

[24] K. G. Wilson, Physical Review D 7, 2911 (1973).

[25] P. Ramond, Field Theory: A Modern Primer (AddisonWesley, Reading, MA, 1989), 2nd ed.

[26] J. Zinn-Justin, Quantum field theory and critical phenomena (Oxford University Press, NY, 1999).

[27] S.-J. Chang and S.-K. Ma, Phys. Rev. 180, 1506 (1969).

[28] M. Altaisky, PEPAN Letters 2, 337 (2005).

[29] J.-P. Eckmann and H. Epstein, Comm. Math. Phys. 64, 95 (1979).

[30] V. Vladimirov, I. Volovich, and E. Zelenov, p-Adic numbers in mathematical physics (World Scientific, Singapore, 1994).

[31] R. Sorkin, Causal sets: Discrete gravity, http://arxiv.org/abs/gr-qc/0309009 (2003).

[32] J. R. Klauder and R. F. Streater, J. Math. Phys. 32, 1609 (1991).

[33] S. Ali, J. Antoine, J. Gazeau, and U. Mueller, Rev. Math. Phys 7, 1013 (1995).

[34] J. Kogut and D. Soper, Phys. Rev. D 1, 2901 (1970).

[35] E. Gorodnitskiy and M. Perel, in Proc. Int. Conf. Days on Difraction 2011, edited by I. Andronov (SPbU, St.Petersburg, 2011), pp. 72-77.

[36] M. Altaisky and N. Kaputkina, Russian Physics Journal
55, 1177 (2013).

[37] M. Perel and M. Sidorenko, J. Phys. A: Math. Theor. 40, 3441 (2007).

[38] L. Faddeev and V. Popov, Phys. Lett. B 25, 29 (1967).

[39] N. Kroll and W. Lamb, Phys. Rev. 75, 388 (1949).

[40] E. Salpeter, Phys. Rev. 87, 328 (1952).

[41] E. Salpeter, Phys. Rev. 89, 93 (1953).

[42] G. Erickson and D. Yennie, Ann. Phys. 35, 271 (1965).

[43] N. N. Bogoliubov and D. V. Shirkov, Introduction to the theory of quantized fields (John Wiley, New York, 1980).

[44] A. Kizilersu, M. Reenders, and M. Pennington, Phys. Rev. D 52, 1242 (1995).

[45] J. Ward, Phys. Rev. 78, 182 (1950).

[46] Y. Takahashi, Nuovo Cimento 6, 371 (1957).

[47] L. H. Ryder, Quantum Field Theory (Cambridge University Press, 1985).

[48] S. Albeverio and M. V. Altaisky, New Advances in Physics 5, 1 (2011), URL arXiv:0901.2806.

[49] G. Battle and P. Federbush, Comm. Math. Phys. 109, 417 (1987).

[50] M. Altaisky and N. Kaputkina, JETP Letters 94, 341 (2011).

[51] A. E. Dorokhov and W. Broniowski, Phys. Rev. D 65, 094007 (2002).

[52] A. Dorokhov, Eur. Phys. J. C 42, 309 (2005).

[53] M. Altaisky, Doklady Physics 51, 326 (2006). 